MINI-SYMPOSIUM

\title{
Closure of patent foramen ovale: technique, piffalls, complications, and follow up
}

\section{B Meier}

Heart 2005;91:444-448. doi: 10.1136/hrt.2004.052258

$\mathrm{T}$ he first atrial septal defects (ASDs) were closed percutaneously in 1974 and reports about their closure published in $1976 .{ }^{1}$ In the 1980s the Rashkind occluder was introduced and revived the interest in the topic, ${ }^{2}$ focusing for the first time on the patent foramen ovale (PFO). ${ }^{3}$ This device is still available in two modified versions. Its initial name, Rashkind Clamshell occluder, has been changed, first into CardioSEAL and more recently into STARFlex (Nitinol Medical Technologies, Boston, Massachusetts, USA). Two other devices are the Sideris Buttoned Device (Custom Medical Devices, Amarillo, Texas, USA) and the Angel Wings device (Microvena Corp, Whitebear Lake, Minnesota, USA), more recently called the Guardian Angel device. The ASDOS device and the Monodisk device are no longer on the market. All these devices had one major problem in common pertaining to the closure of ASDs. They were not selfcentering. Although some efforts were made to remedy this with intricate nitinol wire constructions or interlinking filaments, the only self-centering device was introduced in the 1990s - the Amplatzer ASD occluder. ${ }^{4}$ Figure 1 shows a selection of these devices.

The PFO was initially considered by interventional cardiologists simply as one of the manifestations of an ASD to be left to their paediatric colleagues. However, about 10 years ago, it was realised that the PFO not only represented the most common form of an ASD but that it also exhibited its problems exclusively in adults and represented the easiest target for closure. A derivative of the Clamshell occluder was dedicated to PFO closure and named PFO STAR (Cardia, Burnsville, Minnesota, USA); the Amplatzer occluder was modified for the PFO indication and first implanted on 10 September 1997 by the author in the presence of Kurt Amplatz, the inventor. Like all Amplatzer occluder devices, it consists of a nitinol mesh double disk containing polyester fabric inside the two disks. The disks are connected by a thin neck formed by the woven wires forming the disks. The neck is twisted around its long axis and hence is extendable. The device has to be screwed on to a pusher/puller cable and pulled into an 8 or 9 French introducer sheath. When pushed out of the sheath, it resumes its disk shape instantly. The whole process is fully reversible, as many times as required, up to the moment the device is unscrewed from the pusher/ puller cable.

Three sizes of Amplatzer PFO closure devices are available and named after the diameter of the right sided disk. The most commonly used $25 \mathrm{~mm}$ Amplatzer PFO occluder features a right sided disk of $25 \mathrm{~mm}$ diameter and a left sided disk of $18 \mathrm{~mm}$ diameter. The $18 \mathrm{~mm}$ PFO occluder comprises two $18 \mathrm{~mm}$ disks and is meant for small PFOs with a stable septum primum. The $35 \mathrm{~mm}$ Amplatzer PFO occluder is destined for large PFOs with an extremely redundant and flimsy septum primum (atrial septum aneurysm) and features a $35 \mathrm{~mm}$ disk on the right side and a $28 \mathrm{~mm}$ disk on the left side. It requires a 9 French sheath in contrast to the two smaller devices fitting through an 8
French sheath. The PFO STAR device is made by square Ivalon patches fixed to two nitinol cross bars connected by a $2 \mathrm{~mm}$ metallic axis. The pusher cable is derived from a biotome gripping a ball tip at the right side end of the axis. The two squares are attached in a way that the blunted nitinol wire tips alternate to avoid pressure necrosis of the tissue between two opposed wire ends. The device comes in several sizes. The minimum sheath size is 10 French, the maximum 12 French. More recent generations of these devices feature a hexagon shape.

The Helex septal occluder is the most recently developed of the devices (WL Gore and Associates, Flagstaff, Arizona, USA). It is a hybrid device recommended for PFO and ASD closure. It has a distensible neck in its final shape but also some self-centering qualities. It consists of a nitinol wire with a polytetrafluoroethylene patch attached in a banner-like fashion. Within the delivery sheath it is fully elongated. Once implanted, it forms a double disk (15-35 mm in diameter), one on each side of the septum. The sheath size required for implantation is 9 French.

The most widely used devices so far for PFO closure are the CardioSEAL device in the USA (2000 to 2002 the only one available) and the Amplatzer PFO occluder in the rest of the world (available in the USA since 2002). The CardioSEAL device was the model for the PFO STAR and differs from it in two major aspects. The wires spreading the tissue have joints made of spring coils shaped into the wires, and the release mechanism employs the principle of a ball tip end on the device and a ball tip end on a cable within the pusher. Inserting the ball tip end of the device first into a cylindrical case at the end of the pusher and pulling back the ball tip end of the pusher cable, the ball tip of the device is enclosed in the case until the pusher cable is pushed out of the case first, thereby releasing the ball tip end of the device. The introducer sheaths required for the different sizes of the CardioSEAL or STARFlex devices vary from 10 to 14 French.

\section{IMPLANTATION TECHNIQUE}

Many aspects of the implantation technique pertain to all devices. It is essential that the diagnosis of a PFO is made unequivocally. The gold standard for this is a transoesophageal echocardiogram with a bubble test after a sustained Valsalva manoeuvre. Immediately after the release of the Valsalva manoeuvre, the blood kept out of the thorax during Valsalva rushes back and fills the right atrium before filling the left atrium, thereby opening the PFO. Provided the correct plane is visualised, bubbles can be seen passing through the gaping foramen ovale. The septum secundum positioned towards the aorta is usually a robust, wedge-like structure. The caudad septum primum may be paper thin and extremely mobile. An atrial septal aneurysm is usually diagnosed when the septum primum moves more than $10 \mathrm{~mm}$ into each atrium during certain phases of the cardiac cycle. Aneurysm is a misnomer as it merely describes the mobility of the non-muscular part of the septum primum. 


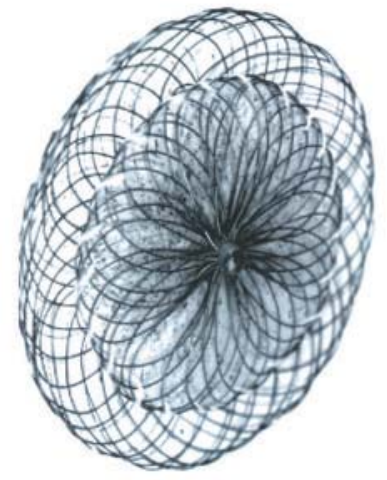

Amplatzer PFO Occluder

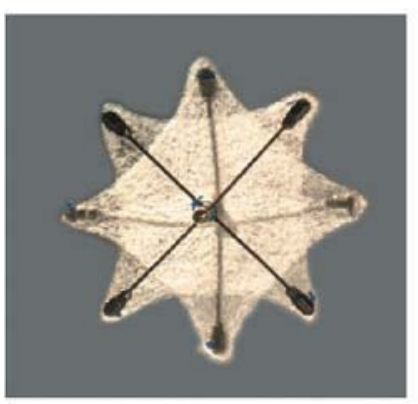

PFO Star

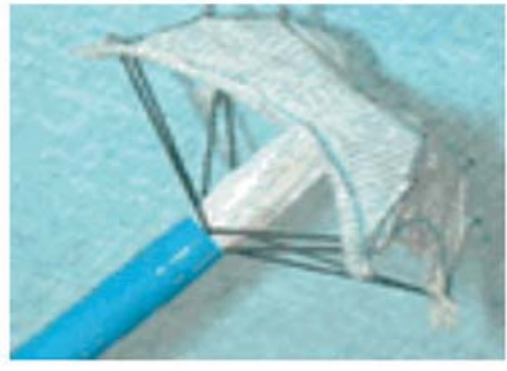

STARFlex

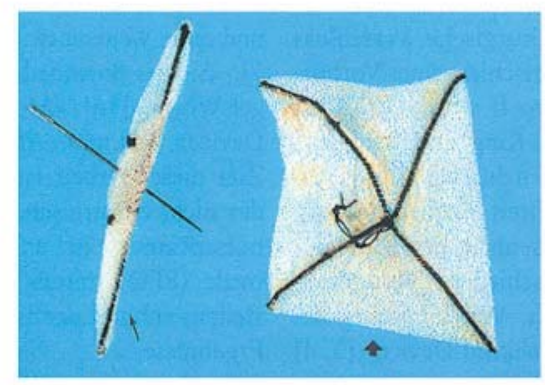

Buttoned Device

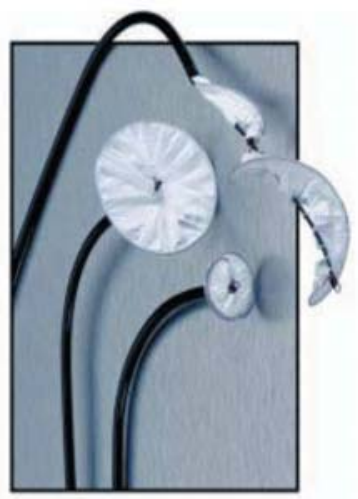

Helex Septal Occluder

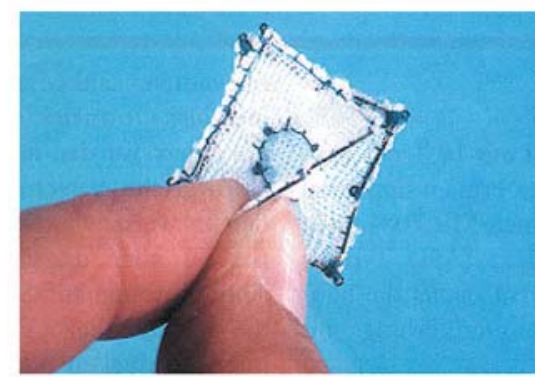

Angel-Wings

Figure 1 Various devices used for percutaneous catheter based patent foramen ovale (PFO) closure.

Nevertheless, the presence of a highly mobile septum primum has been identified as an important risk factor for paradoxical embolism. Initially, this mobility had been considered a risk factor on its own, even in the absence of a PFO. Today this can no longer be supported. ${ }^{5}$ It is now generally accepted that closing the hole is all it takes. Stenting the septum primum with a large device is no longer deemed necessary.

A PFO clearly demonstrated by this transoesophageal echocardiographic technique can be found during catheterisation virtually always by sliding a curved catheter along the septum primum towards the head. This opens the foramen and provides passage. If the passage cannot be found easily (funnel shaped PFO) the manoeuvre is repeated, torquing the catheter as soon as it engages the entrance of the PFO. A wire may be required for further probing in difficult cases. Not infrequently, the initial wire pushed up into the right atrium through the puncturing needle may already find the left atrium in the presence of a widely patent PFO with an atrial septal aneurysm. Rarely, a PFO cannot be found in spite of echocardiographic proof of bubbles passing in that region. There may be two reasons for this. First, the foramen ovale may be fused over almost the entire width of its mouth. If only one corner remains open, the gap may be in an awkward direction for the catheter probing through a funnel shaped entrance of the PFO. An injection of contrast medium through the probing catheter can usually demonstrate the location of the PFO, but it will require an investment of time and material to negotiate such a passage. Moreover it is doubtful that such a small, difficult to find passage would be a significant risk for paradoxical embolism. Second, instead of a PFO a small ASD may be located in the vicinity of a completely fused foramen ovale. If such a small hole is located in a flimsy part of a volatile septum primum it may be as difficult to find as a needle in a haystack and probably irrelevant for paradoxical embolism as well.

The fitting sheath for the selected device is placed over the wire positioned in the left atrium. The obturator of the sheath is withdrawn slowly to avoid aspiration of air during this process. If a sheath without a sidearm is utilised (currently only with the Amplatzer method), the risk of air embolisation during the procedure is small as the sheath can be flushed just like any regular cardiac catheter. If sheaths with side arms are used, purging is much more difficult and incomplete and a certain percentage of clinically manifest air embolism cannot be avoided. This becomes usually apparent by transient ST elevation in the inferior leads. Given the supine position, air bubbles will travel along the ventral side of the left ventricle and the sinus of Valsalva, thereby finding the take-off of the right coronary artery most easily. Only large quantities of air are clinically relevant as they may block flow in both coronary arteries or lead to cerebral symptoms. Persistent sequelae of air embolism during PFO closure have not been described. ${ }^{6}$

The left sided portion of the device is opened in the left atrium, and sheath and device are pulled back as a unit until the open left sided device lies parallel against the interatrial septum, thereby closing the PFO. Under continued tension on the device the sheath is withdrawn further and the right sided part of the device released, embracing the atrial septum. The correct position is assured and the device is released with a final assessment of the device position. Even if the PFO is initially not tight, it may become completely closed, once endocardium has overgrown the device. This may take several weeks to months. 
Debate is ongoing whether echocardiographic guidance is required for percutaneous PFO closure. Transoesophageal echocardiography yields the best picture but it is uncomfortable for the patient lying on his back on the catheterisation table. Only when it is undertaken in combination with intubation and general anaesthesia does it provide a comfortable working environment for the operator without having the patient suffer unnecessarily. However, it turns a relatively simple 15 minute procedure into a demanding intervention for the patient with a prolonged recovery period, not to mention a sore throat.

A more convenient alternative is intracardiac ultrasound. However, this increases the cost of the already expensive device implantation. In addition it requires a second large (12 French) venous access, which increases the risk of an arteriovenous fistula at the puncture site, an already not too rare complication of the procedure, occurring in about $1 \%$ of cases. In our own experience with over 500 PFO closures without echocardiographic guidance, the need to summon echocardiography for assistance in the middle of the procedure has never arisen.

\section{PITFALLS}

Several technical advantages favour the Amplatzer PFO occluder. Although rarely an issue, even after deploying the right sided disk, the device can be easily recaptured into the sheath and placed a second time or removed. This also holds true for the Helex septal occluder which, however, is by far the most difficult to use device requiring a long learning curve. All other devices have to be completely removed outside the body and often replaced by a new one, if the position is unacceptable after releasing the right sided disk. The fact that they are folded like two umbrellas connected at their tip within the delivery sheath explains that pulling the right sided disk back will fold it the wrong way. This must be corrected outside of the body, and the same device can only be re-used if it has not been distorted during the process.

The Amplatzer PFO occluder and the Helex septal occluder are also the only devices that conform to long funnel shaped PFOs. The other devices have a fixed length of the connector between the disks. This usually results in an incomplete opening of the right sided umbrella, which remains partially stuck in a tunnel-like PFO. This may still provide a complete occlusion of the PFO, but the device arms sticking out into the right atrium like a half opened umbrella are not a nice sight for the echocardiographer.

For procedures done without echocardiography, a few anatomical landmarks have to be observed during implantation. Contrast medium injection once the device is deployed but still connected to the pusher cable is possible with all techniques. In a usually left anterior oblique projection with a cranial tilt, the two disks can be seen in profile. They form two lines almost parallel in the inferior part where the membranous part of the septum primum barely divides them. In the superior part they should be gaping at a certain angle as they embrace the wedge-like septum secundum. This has been alluded to by us as the Pacman sign (fig 2), making reference to the well known arcade figure gobbling up a dot. ${ }^{7}$ The contrast medium outlining the right atrium should clearly depict that all of the right sided disk and none of the left sided disk is in the right atrium. A residual shunt into the left atrium seen at that moment is of little importance for the reasons stated above. A final check before release is usually done by wiggling on the pusher cable. On an extremely rare occasion, the device may be dislocated by such wiggling. In that case it should be replaced by a larger device.

\section{COMPLICATIONS}

Observing these precautions, embolisation of the device during or after implantation has not occurred in our experience of several hundred consecutive cases. Embolisation remains, however, one of the possible complications. ${ }^{6}$ Other serious complications occurring in less than $1 \%$ of cases are infection, erosion into the pericardium or aorta at the rim of the device, a new ASD caused by the lower rim tearing the thin septum primum, or paroxysmal or persistent atrial fibrillation. Some palpitations are not uncommon and are reported by about $10 \%$ of patients in the early period after implantation. They usually subside spontaneously.

Thrombosis on the device has been found in about $6 \%$ of devices used for PFO closures at the one month transoesophageal echocardiographic control in 1000 patients, except for the Amplatzer PFO occluder where it was found in less than $1 \%{ }^{8}$ A recent study reported thrombosis on five of 23 CardioSEAL devices checked at one month but in none of 20 Amplatzer PFO occluders. ${ }^{9}$

\section{CLINICAL RESULTS}

It can be assumed that clinical results will be better with devices providing a higher complete closure rate. ${ }^{10}$ Our initial experience with 170 patients treated with five different devices and a follow up of about seven years showed an incidence of recurrent embolic events such as transient ischaemic attacks, cerebral vascular attacks, or peripheral embolism of $2 \%$ per year. This contrasts favourably with the literature on the natural course of patients with a PFO and a cryptogenic stroke with an incidence of recurrences of 5\% per year. ${ }^{11}$ It contrasts even more favourably with 42 patients on

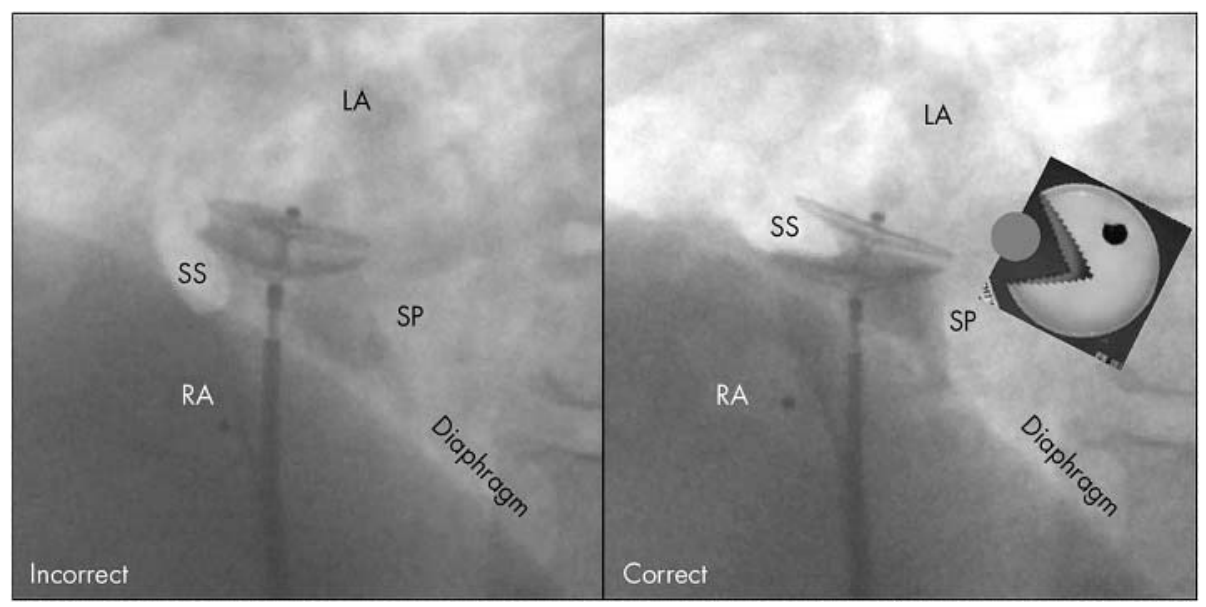

Figure 2 Angiographic landmarks for adequate placement of an Amplatzer PFO occluder before release from the pusher cable. The left panel depicts an incorrect position with both $18 \mathrm{~mm}$ disks on the left (upper) side of the tongue-like septum secundum (SS) that is, in the left atrium (LA). The right panel shows the correct position attained after pulling the right disk back into the introducer sheath and redeploying it with more tension on the pusher cable. The thick septum secundum now lies between the two disks with the lower disk entirely in the right atrium (RA). The insert shows the arcade figure "Pacman" gobbling up a dot and providing the name Pacman sign in analogy. ${ }^{7} \mathrm{SP}$, septum primum. 


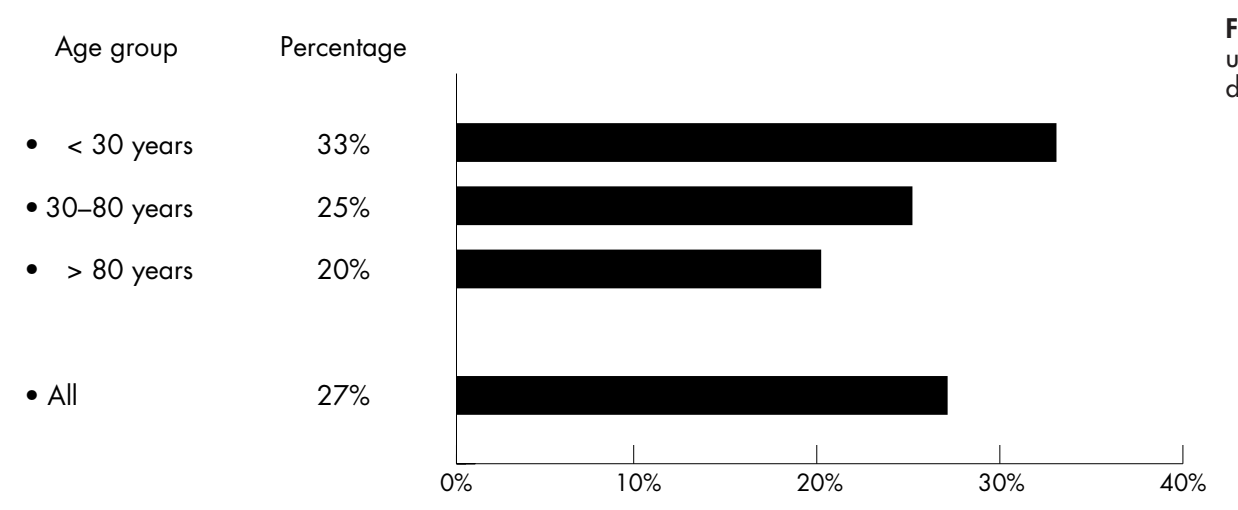

Figure 3 Presence of a PFO in 965 unselected necropsies of all cause deaths at various ages. ${ }^{17}$

warfarin and 56 patients on aspirin during a two year follow up after a cryptogenic stroke in the presence of a PFO with a recurrence rate of death or stroke (transient ischaemic attacks not included) of $5 \%$ and $9 \%$ per year, respectively. ${ }^{12}$

Pooling the results of the published studies on percutaneous PFO closure, it appears that the intervention yields favourable clinical results over a conservative treatment. ${ }^{6}$ Randomised trials, however, are not yet available. Three of them are on the way. The PC trial, an international multicentre trial with the Amplatzer PFO occluder, started recruiting patients about three years ago but has yet to reach the target of 425 patients. The RESPECT trial, which is also using the Amplatzer PFO occluder exclusively, has started recruiting the planned 300 patients late in 2003, as has the CLOSURE trial using the CardioSEAL device with an inclusion target of 1600 patients. These two trials are multicentre trials in the USA. What comes closest to a randomised trial is a contemporary matched control follow up study in about 300 patients, of which half were arbitrarily sent for PFO closure by neurologists and half were treated conservatively by the same physicians. Already at a follow up of four years, there was a trend in favour of device closure with an average of 5\% events per year (counting all neurological or peripheral symptoms) compared with 7\% in the conservative group. This advantage was significant in terms of major strokes which only occurred in the conservative group. It was also significant in the subgroup of patients who had had more than two events before treatment allocation as well as in those who had complete closure at the six month follow up transoesophageal echocardiography in the device group. ${ }^{13}$

\section{SURGICAL CLOSURE}

Comparing clinical results of device closure with surgical results, a certain similarity can be observed. ${ }^{14}$ Considering the inconvenience of heart surgery (general anaesthesia, scar, prolonged hospital stay, wound infection, etc), and the fact that not a single emergency operation following a failed attempt of device closure for PFO has been described even though all patients could have undergone uncompromised surgery in case of failure, it appears imperative that device closure is the therapy of first choice.

\section{NEW EMERGING INDICATIONS FOR PFO CLOSURE}

The clinical justification for PFO closure after paradoxical embolism is still lacking. Other indications are even less supported by clinical data. Nonetheless, growing interest has been observed recently in the connection between the PFO and diving accidents ${ }^{15}$ or migraine. ${ }^{16}$

\section{OUTLOOK}

The indication for PFO closure in the face of paradoxical embolism has been widely accepted in the presence of an atrial septal aneurysm or after several embolic events. It has been adopted at our centre for any suspicion of paradoxical embolism. This has introduced a frequency of PFO closure at our catheterisation laboratory that already amounts to more than $10 \%$ of the entire interventional activity. This is bound to increase further, particularly if divers and patients with migraine are included. Finally, a scenario can be envisioned where the PFO is considered a threat for longevity. A pathological study on about 1000 necropsies of all ages showed that the prevalence of PFO decreased from $33 \%$ in those younger than 30 years of age to $20 \%$ in those over 80 (fig 3). ${ }^{17}$ Late spontaneous closure of a PFO is a likely explanation, but not less likely is a selective mortality of PFO carriers. Percutaneous PFO closure is the most simple procedure in interventional cardiology. Further simplified and ubiquitously available it could be advocated as a kind of mechanical vaccination against the late hazards of a PFO. It would have to be carried out in teenagers screened for a PFO. Before that age, venous thrombosis is virtually unheard of and the PFO has no potential for harm.

Correspondence to: Professor Bernhard Meier, Swiss Cardiovascular Centre Bern, University Hospital Bern, 3010 Bern, Switzerland; bernhard.meier@insel.ch

\section{REFERENCES}

1 King TD, Thompson SL, Steiner C, et al. Secundum atrial septal defect. Nonoperative closure during cardiac catheterization. JAMA 1976;235:2506-9.

2 Rashkind WJ. Transcatheter treatment of congenital heart disease. Circulation 1983;67:711-16.

3 Bridges ND, Hellenbrand W, Latson L, et al. Transcatheter closure of patent foramen ovale after presumed paradoxical embolism. Circulation 1992;86:1902-8.

4 Sharafuddin MJ, Gu X, Titus JL, et al. Transvenous closure of secundum atrial septal defects: preliminary results with a new self-expanding nitinol prosthesis in a swine model. Circulation 1997:95:2162-8.

5 Mas JL, Arquizan C, Lamy C, et al. Recurrent cerebrovascular events associated with patent foramen ovale, atrial septal aneurysm, or both. N Engl J Med 2001;345:1740-6.

6 Khairy P, O'Donnell CP, Landzberg MJ. Transcatheter closure versus medical therapy of patent foramen ovale and presumed paradoxical thromboemboli: a systematic review. Ann Intern Med 2003;139:753-60.

7 Meier B. Pacman sign during device closure of the patent foramen ovale. Catheter Cardiovasc Interv 2003:60:221-3.

8 Krumsdorf U, Ostermayer S, Billinger K, et al. Incidence and clinical course of thrombus formation on atrial septal defect and patient foramen ovale closure devices in 1,000 consecutive patients. J Am Coll Cardiol 2004:43:302-9.

9 Anzai H, Child J, Natterson B, et al. Incidence of thrombus formation on the CardioSeal and the Amplatzer interatrial closure devices. Am J Cardiol 2004;93:426-36. 
10 Schwerzmann $M$, Windecker S, Wahl A, et al. Percutaneous closure of patent foramen ovale: impact of device design on safety and efficacy. Heart 2004;90:186-90.

11 De Castro S, Cartoni D, Fiorelli M, et al. Morphological and functional characteristics of patent foramen ovale and their embolic implications. Stroke 2000;31:2407-13.

12 Mohr JP, Thompson JLP, Lazar RM, et al, for the Warfarin-Aspirin Recurrent Stroke Study Group. A comparison of warfarin and aspirin for the prevention of recurrent ischemic stroke. New Engl J Med 2001;345:1444-51.

13 Windecker S, Wahl A, Nedeltchev K, et al. Comparison of medical treatment with percutaneous closure of patent foramen ovale in patients with cryptogenic stroke. J Am Coll Cardiol 2004;44:750-8.
14 Dearani JA, Ugurlu BS, Danielson GK, et al. Surgical patent foramen ovale closure for prevention of paradoxical embolism-related cerebrovascular ischemic events. Circulation 1999;100(19 suppl):II171-5.

15 Schwerzmann M, Seiler C, Lipp E, et al. Relation between directly detected patent foramen ovale and ischemic brain lesions in sport divers. Ann Intern Med 2001; 134:21-4.

16 Wilmshurst PT, Nightingale S, Walsh KP, et al. Effect on migraine of closure of cardiac right-to-left shunts to prevent recurrence of decompression illness or stroke or for haemodynamic reasons. Lancet 2000;356:1648-51.

17 Hagen PT, Scholz DG, Edwards WD. Incidence and size of patent foramen ovale during the first 10 decades of life: an autopsy study of 965 normal hearts. Mayo Clin Proc 1984;59:17-20.

\section{IMAGES IN CARDIOLOGY}

\section{Accelerated atherosclerosis following intracoronary haematopoietic stem cell administration}

$\mathrm{H}$ aematopoietic stem cell therapy is emerging as a promising treatment for patients with left ventricular (LV) dysfunction after acute myocardial infarction. However, recent experimental and clinical data suggest accelerated atherosclerosis occurs following bone marrow mobilisation or intracoronary haematopoietic stem cell therapy. We report on a 74 year old man with acute anterior myocardial infarction treated with primary percutaneous coronary intervention and stenting of the proximal left anterior descending coronary artery (LAD) with a paclitaxel eluting stent (Taxus, Boston Scientific, panel A). Selected haematopoietic $\mathrm{CD} 33^{+}$cells were administered intracoronarily seven days later. Follow up coronary angiography at four months showed no restenosis at the site of the stenting.
In contrast, a critical mid LAD lesion, associated with a significant decrease in the myocardial fractional flow reserve, was noted distal to the stent (panel B). LV ejection fraction showed a mild increase from $46 \%$ to $51 \%$ at follow up angiography.

Accelerated atherosclerosis following intracoronary stem cell therapy may cloud myocardial recovery and requires careful assessment in further studies.

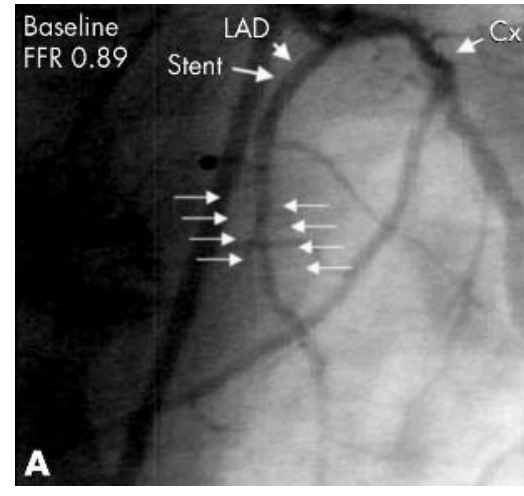

Baseline coronary angiogram before CD133+ cell injection. Multiple arrows indicate diffuse atherosclerosis in the mid LAD distal to the stent deployment. FFR, myocardial fractional flow reserve; $L A D$, left anterior descending coronary artery; CX, left circumflex coronary artery.

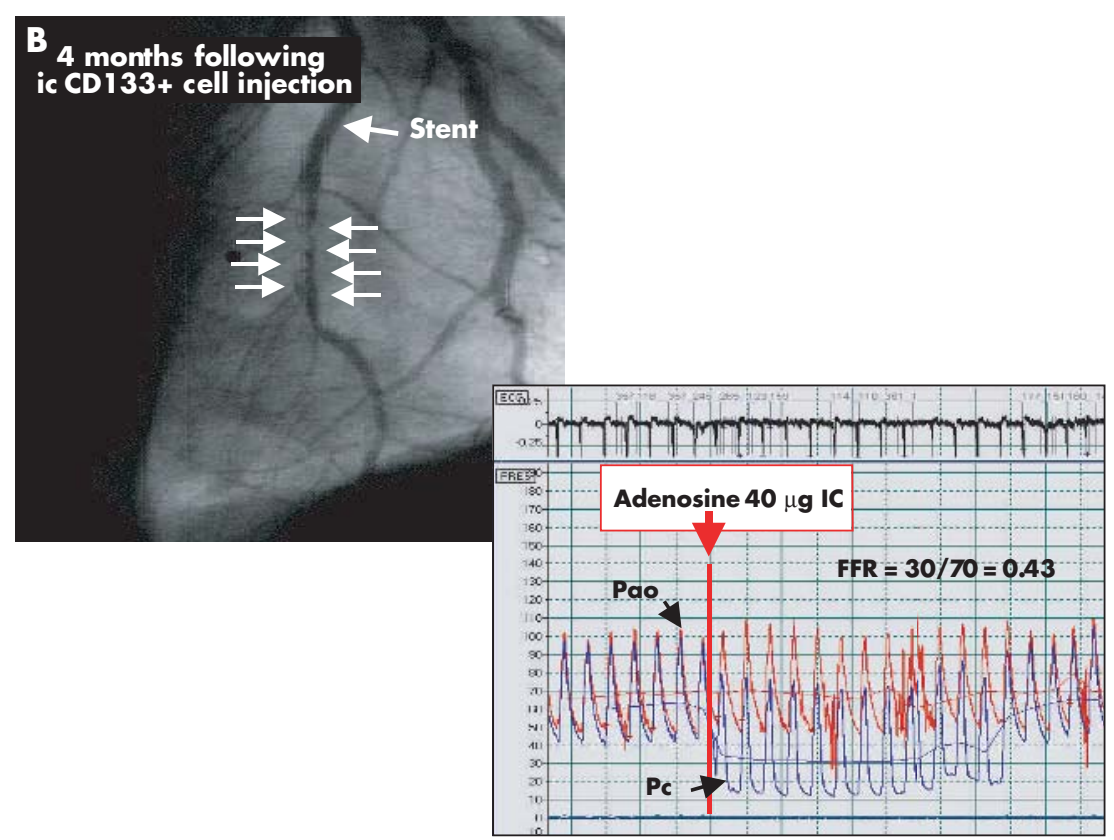

Upper panel shows coronary angiography at four months follow up. Arrows indicate mid LAD with the progression of the coronary atherosclerosis with de novo critical lesion. Lower panel shows pressure measurements with myocardial fractional flow reserve. Pao, aortic pressure; Pc, distal coronary pressure. 